\title{
Global initiative targets progressive multiple sclerosis
}

\author{
Goal to develop treatments for a refractory form of the disease.
}

\section{Elie Dolgin}

17 September 2014

\section{BOSTON, MASSACHUSETTS}

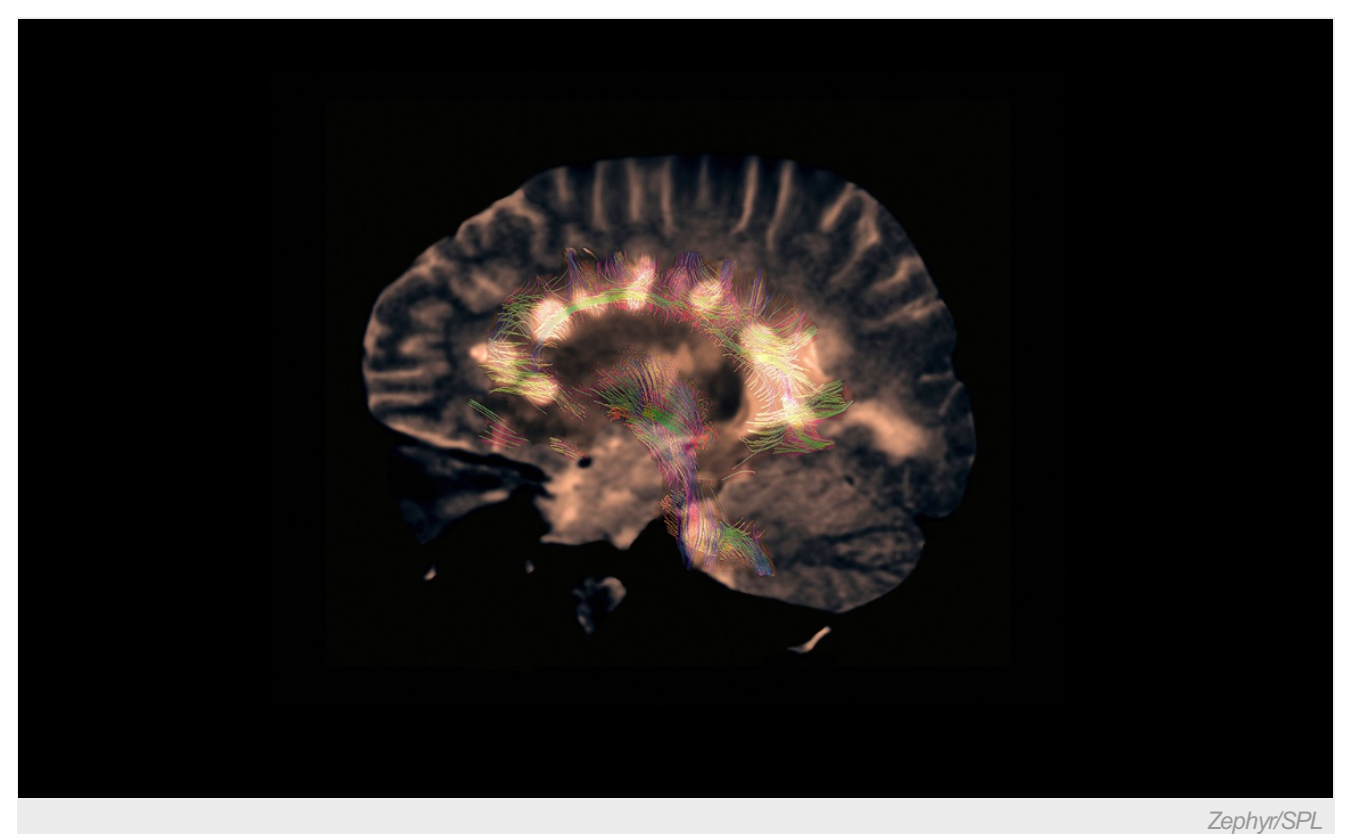

New drugs for multiple sclerosis are of no use in treating the disease's progressive form.

When Carol Steinberg was diagnosed with multiple sclerosis (MS) in 1995, there was only one drug approved by the US Food and Drug Administration to treat the disease. Now there are eleven.

Yet none of these agents can help Steinberg. She suffers from progressive MS, a form of the disease that is characterized by steadily worsening neurological function. All eleven approved drugs combat the unpredictable symptom outbreaks that are associated with the relapsing-remitting form of MS. Around $85 \%$ of newly diagnosed patients have the relapsing-remitting form; after 10 to 20 years, most of them develop the progressive type.

The lack of good treatment options for progressive MS weighs heavily on Steinberg. She uses a wheelchair, but continues to work as a trial lawyer in Newton, Massachusetts. "I'm constantly afraid of my disease getting worse," she says.

A global initiative called the Progressive MS Alliance now hopes to kick-start the development of therapies specifically for Steinberg and the million or so people worldwide living with progressive MS. On 11 September, at a joint meeting of the European and Americas Committees for Treatment and Research in Multiple Sclerosis, the alliance announced an inaugural round of research awards - part of a six-year, €22-million (US\$28.5-million) programme that is the first concerted effort to tackle the disease's less-common form.

"We need a new focus on progressive MS," says Neil Scolding, a specialist in MS at the University of Bristol, UK, who is not involved in the initiative. "This alliance will provide a huge stimulus — not only in terms of the research it will fund, but in terms of raising the profile of progressive MS and in helping the pharmaceutical industry realize what a priority this is."

\section{Treatment void}

All approved MS drugs work by combating the inflammation that occurs when the body's immune cells attack the nervous system — the primary cause of relapsing-remitting MS. Yet, unlike relapsing-remitting disease, inflammation has only a minor role in progressive MS, in which steady nerve degeneration is the major pathological factor. Some researchers have even questioned whether progressive MS is one end of a wide spectrum or a separate disease entity. 
No medications seem to make a dent in the physical declines experienced by the $10-15 \%$ of patients with MS whose disease progression is continuous from diagnosis. And only one drug is approved to help patients who develop progressive MS after first experiencing relapsing-remitting disease. But this agent, an immunosuppressant originally developed to treat certain forms of cancer, comes with such harsh side effects, particularly on the heart, that most doctors are loath to prescribe it.

\section{Ambitious aim}

The 22 pilot grants unveiled last week at the Boston meeting are designed to fill knowledge and infrastructure gaps in scientists' understanding of progressive MS. Some grants involve studying genetics, biomarkers or responses to experimental treatments in human patients. For example, Anders Svenningsson, a neurologist at Umeå University in Sweden, received funding to determine whether administering a drug called rituximab directly into the spinal cord could be effective. In past trials, injections into the bloodstream have not been beneficial.

Other projects are focused on preclinical studies of nerve degeneration. In this study area, Robin Avila and her colleagues at Renovo Neural, a contract research company in Cleveland, Ohio, won a $€ 75,000$ award to develop mice with chemically induced progressive disease. "We hope to achieve this progressive MS-like phenotype," Avila says, "and then we can use this model to demonstrate preclinical efficacy of potential therapeutics."

To build on findings from this first funding round, the Progressive MS Alliance - which is led by non-profit MS societies from North America, Europe and Australia - plans to put out a call in November for applications to build international collaborative networks. Each of these will receive multimillion-euro grants for drug-discovery research, biomarker validation or proof-of-concept clinical trials. The ultimate goal, says Giancarlo Comi, a neurologist at the San Raffaele Scientific Institute in Milan, Italy, and co-chair of the alliance's scientific steering committee, is to have a drug in mid- to late-stage clinical testing by the time the alliance's funding runs out in 2019.

"This is a very ambitious aim," Comi admits. "But only if you have ambition can you reach targets."

Nature | doi:10.1038/nature.2014.15948 